University of New Orleans

ScholarWorks@UNO

7-1999

\title{
Differential reflection phase shift under conditions of attenuated internal reflection
}

\author{
R. M.A. Azzam \\ University of New Orleans, razzam@uno.edu
}

Follow this and additional works at: https://scholarworks.uno.edu/ee_facpubs

Part of the Electrical and Electronics Commons, and the Optics Commons

\section{Recommended Citation}

R. M. A. Azzam, "Differential reflection phase shift under conditions of attenuated internal reflection," J. Opt. Soc. Am. A 16, 1700-1702 (1999)

This Article is brought to you for free and open access by the Department of Electrical Engineering at ScholarWorks@UNO. It has been accepted for inclusion in Electrical Engineering Faculty Publications by an authorized administrator of ScholarWorks@UNO. For more information, please contact scholarworks@uno.edu. 
R. M. A. Azzam

\title{
Differential reflection phase shift under conditions of attenuated internal reflection
}

\author{
R. M. A. Azzam \\ School of Sciences and Engineering, The American University in Cairo, P. O. Box 2511, Cairo 11511, Egypt
}

Received October 9, 1998; accepted January 12, 1999

\begin{abstract}
The angle-of-incidence dependence of the differential reflection phase shift $\Delta$ between $p$ and $s$ polarizations is considered a function of the real and imaginary parts of the relative complex dielectric function $\varepsilon$ of an interface in the domain of fractional optical constants, i.e., under conditions of internal reflection. The constraint on complex $\varepsilon$ such that oscillatory and monotonic angular responses are obtained is determined. A sensitive and stable technique, which is based on attenuated internal reflection ellipsometry between the Brewster angle and the critical angle, is proposed for measuring small induced absorption $\left(\varepsilon_{i} \sim 10^{-5}\right)$ in the medium of refraction. (C) 1999 Optical Society of America [S0740-3232(99)01407-6]
\end{abstract}

OCIS codes: 240.0240, 120.0120 .

\section{INTRODUCTION}

When a plane wave of monochromatic light is incident at the planar interface between two homogeneous, optically isotropic, nonmagnetic media from the side of low index of refraction (i.e., in external reflection), the differential reflection phase shift (or ellipsometric angle) $\Delta$ decreases monotonically from $\pi$ to 0 as the angle of incidence $\phi$ is increased from 0 (normal incidence) to $90^{\circ}$ (grazing incidence), respectively. ${ }^{1,2}$ This assumes the presence of absorption in the medium of refraction, the $\exp (j \omega t)$ dependence for time-harmonic wave fields, and the rest of the Nebraska-Muller conventions. ${ }^{3}$

Under conditions of attenuated internal reflection (AIR), the $\Delta$-versus- $\phi$ curve can be oscillatory, exhibiting one minimum and one maximum between normal and grazing incidence. ${ }^{4,5}$ In this paper we determine the constraint on the relative complex dielectric function

$$
\varepsilon=\varepsilon_{1} / \varepsilon_{0}=\varepsilon_{r}-j \varepsilon_{i}
$$

such that either monotonic or oscillatory phase-versusangle response is obtained in AIR (i.e., in the domain of fractional optical constants). In Eq. (1) $\varepsilon_{0}$ and $\varepsilon_{1}$ are the dielectric constants of the media of incidence and refraction, respectively, at a given wavelength.

We also propose the use of AIR ellipsometry between the Brewster angle and the critical angle as a sensitive technique for measuring small absorption in the medium of refraction, as represented by small values of $\varepsilon_{i}$. Measurement of the refractive index on the basis of total internal reflection has received renewed interest recently. 6,7

\section{MONOTONIC AND OSCILLATORY PHASE-VERSUS-ANGLE RESPONSES}

The ratio $\rho=r_{p} / r_{s}$ of the complex-amplitude reflection coefficients for $p$ and $s$ linear polarizations, parallel and perpendicular to the plane of incidence, respectively, is given by ${ }^{1}$

$$
\rho=\frac{\left[\sin \phi \tan \phi-\left(\varepsilon-\sin ^{2} \phi\right)^{1 / 2}\right]}{\left[\sin \phi \tan \phi+\left(\varepsilon-\sin ^{2} \phi\right)^{1 / 2}\right]},
$$

and $\Delta$ is obtained from $\rho$ by

$$
\Delta=\arg (\rho) .
$$

For a given value of $\varepsilon_{r}$ in the range $0 \leqslant \varepsilon_{r} \leqslant 1$, the $\Delta$-versus- $\phi$ curve depends on $\varepsilon_{i}$. As an example, Fig. 1 shows a family of such curves for $\varepsilon_{r}=0.6$. The response is oscillatory for $\varepsilon_{i}=0,0.005,0.015,0.045,0.075$, and 0.10 (curves marked $\mathrm{o}, \mathrm{a}, \mathrm{b}, \mathrm{c}, \mathrm{d}$, and e, respectively) and is monotonic for $\varepsilon_{i}=0.1366,0.2$, and 2.0 (curves $\mathrm{f}, \mathrm{g}$, and $\mathrm{h}$, respectively). The limiting value $\varepsilon_{i}=0.1366$ (curve f) is determined by numerical iteration as the upper bound on $\varepsilon_{i}$, below which an oscillatory response would occur. Figure 2 gives a clear three-dimensional view of the tran-

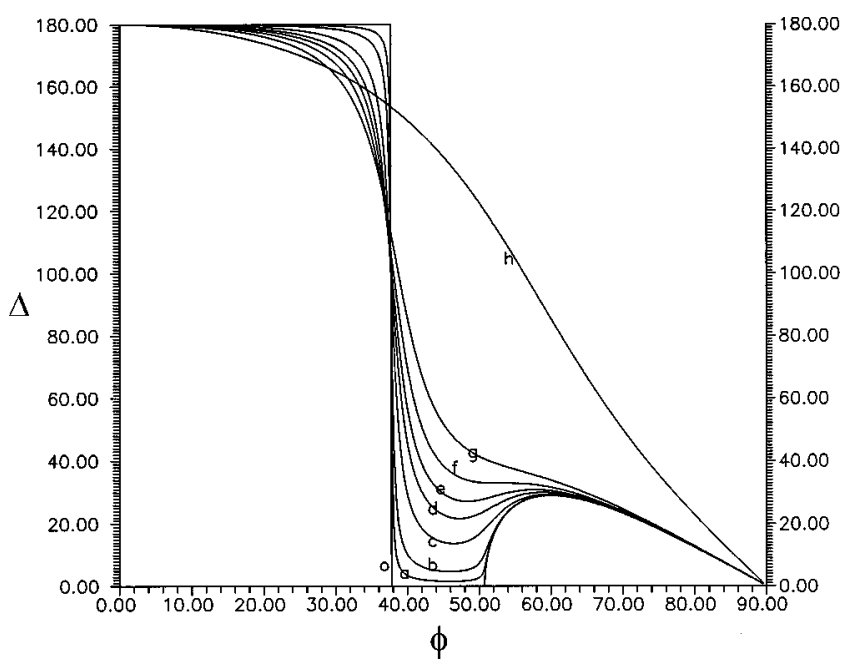

Fig. 1. Differential reflection phase shift $\Delta$ versus angle of inci dence $\phi$ for different values of the imaginary part of the relative dielectric constant $\varepsilon_{i}$ and the constant real part $\varepsilon_{r}=0.6$. The curves marked $\mathrm{o}, \mathrm{a}, \mathrm{b}, \mathrm{c}, \mathrm{d}, \mathrm{e}, \mathrm{f}, \mathrm{g}$, and $\mathrm{h}$ correspond to $\varepsilon_{i}=0$, $0.005,0.015,0.045,0.075,0.1,0.1366,0.2$, and 2.0 , respectively. 


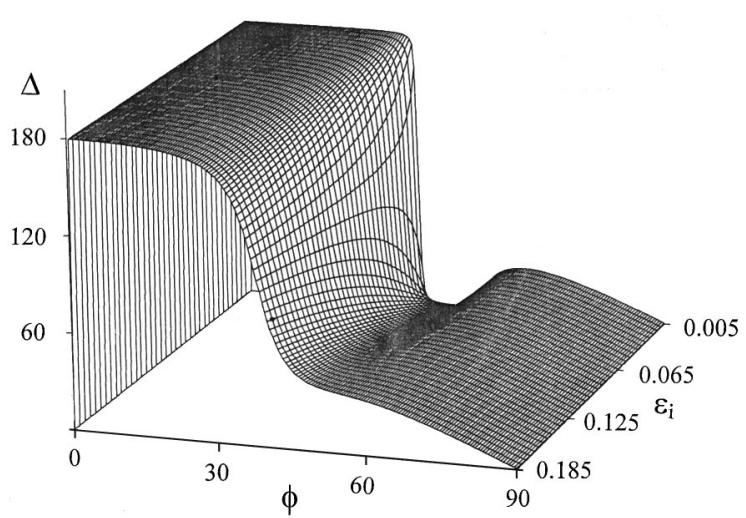

Fig. 2. Differential reflection phase shift $\Delta$ as a function of the angle of incidence $\phi$ and the imaginary part of the relative dielectric constant $\varepsilon_{i}$ for a given value of the real part $\varepsilon_{r}=0.6$.

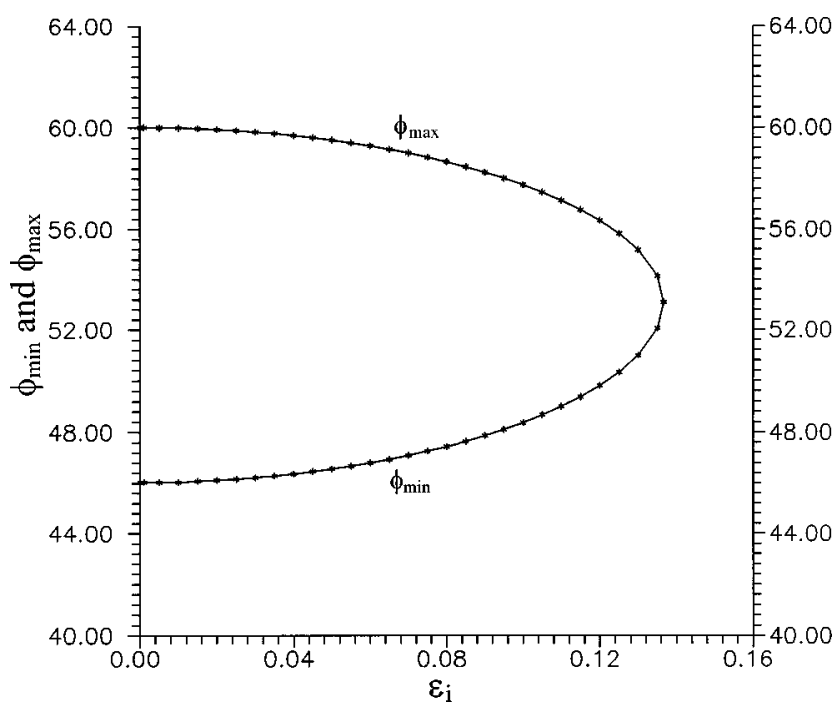

Fig. 3. Angles of incidence $\phi_{\min }$ and $\phi_{\max }$ for minimum and maximum differential reflection phase shift $\Delta$ as functions of the imaginary part of the relative dielectric constant $\varepsilon_{i}$ when $\varepsilon_{r}$ $=0.6$, corresponding to the data shown in Fig. 1 .

sition from oscillatory to monotonic $\Delta$-versus- $\phi$ response as $\varepsilon_{i}$ is increased, for a constant value of $\varepsilon_{r}=0.6$.

In Fig. 1 the range of $\phi$ for which $\Delta=0$, when $\varepsilon_{r}=0.6$ and $\varepsilon_{i}=0$ (curve o for a transparent interface), extends from the Brewster angle $\phi_{B}=\arctan \left(0.6^{1 / 2}\right)=37.76^{\circ}$ to the critical angle $\phi_{c}=\arcsin \left(0.6^{1 / 2}\right)=50.77^{\circ}$. In Fig. 3, for each value of $\varepsilon_{i}$ in the range $0<\varepsilon_{i}<0.1366$, the angles of incidence $\phi_{\min }$ and $\phi_{\max }$ of minimum and maximum differential reflection phase shift $\Delta$ are calculated and are plotted as functions of $\varepsilon_{i}$. The two angles merge, $\phi_{\min }=\phi_{\max }$, at $\varepsilon_{i}=0.1366$. In Fig. 4 the corresponding $\Delta_{\min }$ and $\Delta_{\max }$ are shown as functions of $\varepsilon_{i}$.

By varying $\varepsilon_{r}$ and by repeating the calculation for the upper bound on $\varepsilon_{i}$ for each value of $\varepsilon_{r}$, we obtain the domain of fractional optical constants $\left(\varepsilon_{r}, \varepsilon_{i}\right)$ for which the $\Delta$-versus- $\phi$ response is oscillatory. The results are represented in Fig. 5 by the region of the complex $\varepsilon$ plane between the curve and the $\varepsilon_{r}$ axis. A monotonic response is obtained for all $\left(\varepsilon_{r}, \varepsilon_{i}\right)$ pairs that correspond to points outside this region.

\section{ATTENUATED INTERNAL REFLECTION ELLIPSOMETRY BETWEEN THE BREWSTER ANGLE AND THE CRITICAL ANGLE}

For internal reflection at a sharp interface between two transparent media, $\Delta=0$ between the Brewster angle and the critical angle, as shown in Fig. 1 for $\varepsilon_{r}=0.6$ and $\varepsilon_{i}=0$. If absorption is introduced into the medium of refraction (e.g., by dissolving an absorbing species into an aqueous solution), $\varepsilon_{i}$ is no longer 0 and $\Delta \neq 0$.

From Figs. 1 and 2 it is apparent that measurement of $\Delta$ by internal reflection ellipsometry between the Brewster angle and the critical angle provides a sensitive and stable technique for measuring small values of $\varepsilon_{i}$. Although the sensitivity of $\Delta$ with respect to $\varepsilon_{i}$ is very high at and in the immediate neighborhood of the Brewster and critical angles, the associated measurement is highly susceptible to small angle-of-incidence errors. Therefore ellipsometry at these special angles is not recommended.

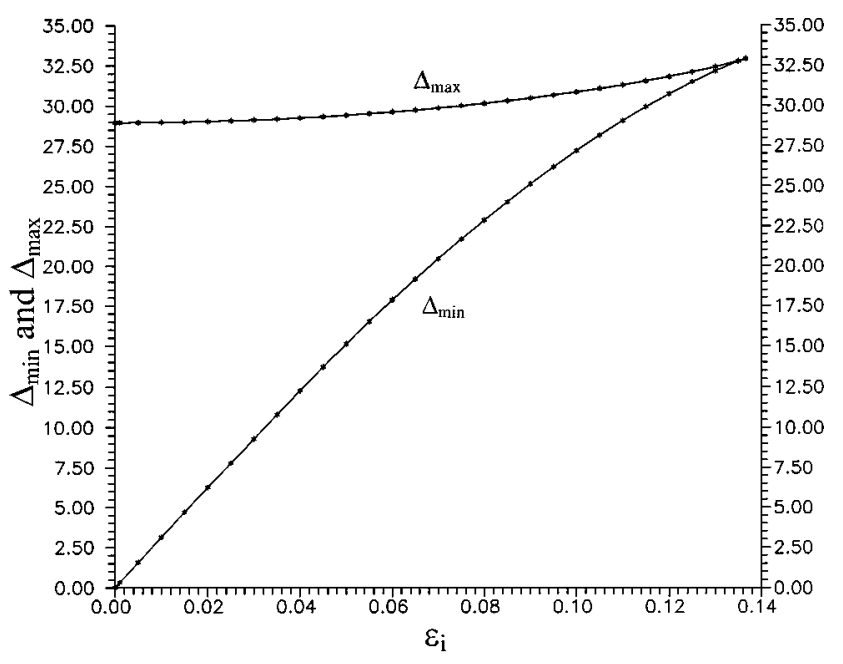

Fig. 4. Minimum and maximum differential reflection phase shift $\Delta_{\min }$ and $\Delta_{\max }$ as functions of the imaginary part of the relative dielectric constant $\varepsilon_{i}$ when $\varepsilon_{r}=0.6$, corresponding to the data shown in Fig. 1.

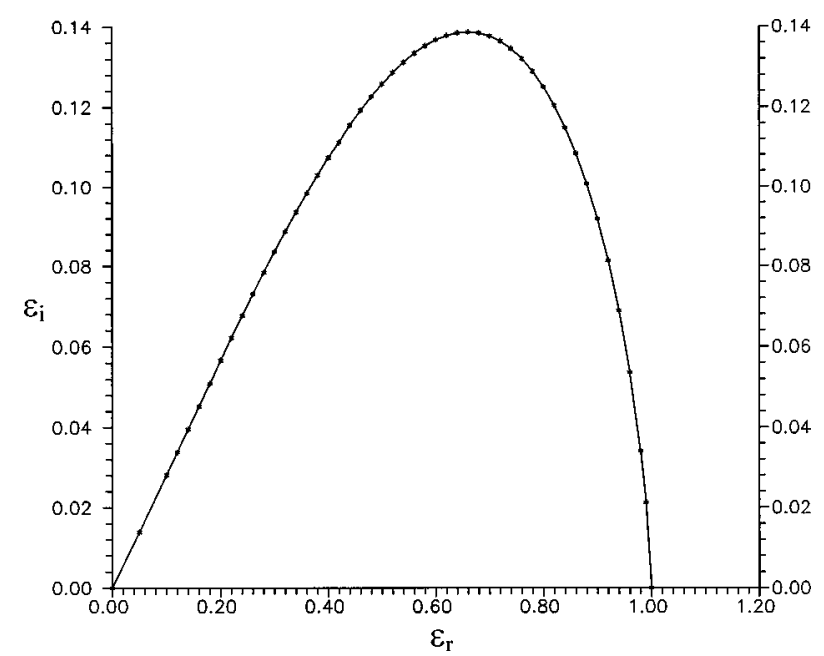

Fig. 5. Upper bound on the imaginary part of the relative dielectric constant $\varepsilon_{i}$ for an oscillatory $\Delta$-versus- $\phi$ response as a function of the real part $\varepsilon_{r}$. 


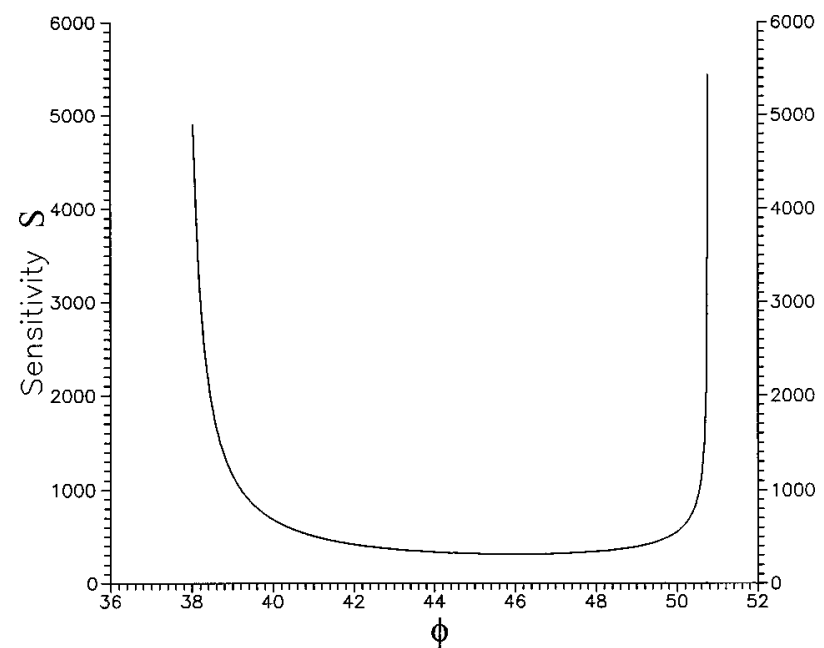

Fig. 6. Ellipsometric sensitivity $S$, Eq. (5), as a function of the angle of incidence $\phi$ for attenuated internal reflection between the Brewster angle and the critical angle of a transparent interface with $\varepsilon_{r}=0.6$

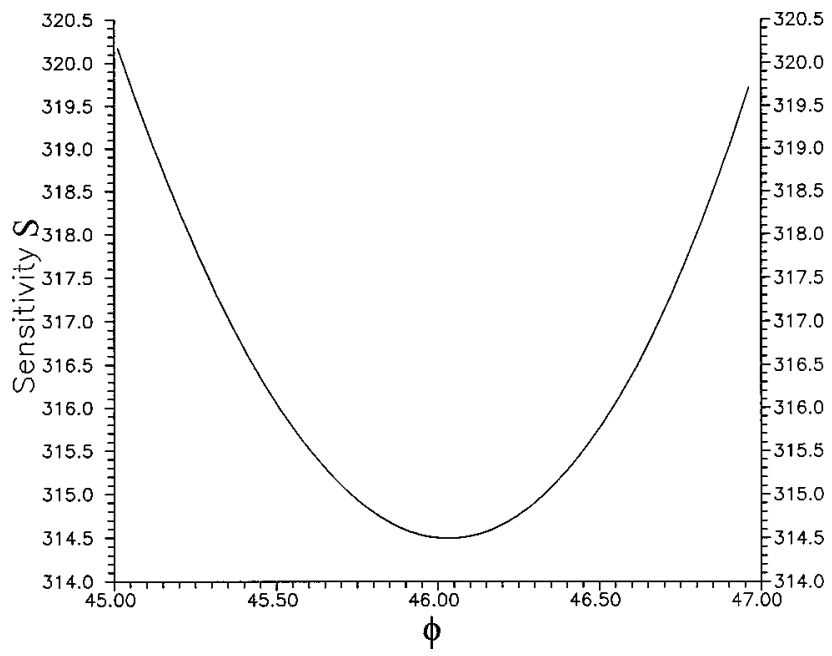

Fig. 7. Same as in Fig. 6, but for a range of $\pm 1^{\circ}$ of the incidence angle $\phi$ around the minimum.

It is instructive to define a sensitivity function as

$$
S=\left[\partial \Delta / \partial \varepsilon_{i}\right]_{\varepsilon_{i}=0} .
$$

From Eqs. (2) and (3), one obtains an explicit expression for $S$ :

$$
\begin{aligned}
S= & \left(180^{\circ} / \pi\right) \sin \phi \tan \phi /\left[\left(\varepsilon_{r}-\sin ^{2} \phi\right)^{1 / 2}\right. \\
& \left.\times\left(\tan ^{2} \phi-\varepsilon_{r}\right)\right] .
\end{aligned}
$$

Equation (5) predicts singularities of the sensitivity function at the Brewster and critical angles. Figure 6 shows the variation of $S$ with $\phi$ between these two angles for $\varepsilon_{r}=0.6$. The sensitivity is minimum at $\phi=46.036^{\circ}$, and Fig. 7 shows $S(\phi)$ within $\pm 1^{\circ}$ of this angle. The minimum sensitivity of 314.49 (degrees per unit change of $\varepsilon_{i}$ ) is quite high. Operation at or near the minimum of $S$ is insensitive to small angle-of-incidence (or beam convergence/divergence) errors. Because changes of $\Delta$ of $0.03^{\circ}$ are readily detectable in ellipsometry, ${ }^{1}$ changes of $\varepsilon_{i}$ from 0 to $10^{-5}$ should be measurable. Notice, however, that the proposed model assumes that the change of $\Delta$ is due to bulk absorption in the medium of refraction and not to the formation of a third thin-film phase at the boundary between the two media. Determination of complex refractive indices by attenuated total reflectance (not phase) measurements were previously reported..$^{8,9}$

\section{CONCLUSION}

In this paper we have found the conditions under which the differential phase shift on reflection at an interface between two media can become an oscillatory function of the angle of incidence. We have also proposed the use of attenuated internal reflection ellipsometry between the Brewster angle and the critical angle as a sensitive and stable technique for measuring the small imaginary part of the complex dielectric function of an absorbing medium of refraction.

The author is on sabbatical leave from the Department of Electrical Engineering, University of New Orleans, New Orleans, Louisiana 70148.

\section{REFERENCES}

1. R. M. A. Azzam and N. M. Bashara, Ellipsometry and Polarized Light (North-Holland, Amsterdam, 1987).

2. R. M. A. Azzam and A. M. El-Saba, "Maximum rate of change of the differential reflection phase shift with respect to the angle of incidence for light reflection at the surface of an absorbing medium," Appl. Opt. 28, 1365-1368 (1989); erratum, Appl. Opt. 35, 213 (1996).

3. R. H. Muller, "Definitions and conventions in ellipsometry," Surf. Sci. 16, 14-33 (1969).

4. R. M. A. Azzam, "Contours of constant principal angle and constant principal azimuth in the complex plane," J. Opt. Soc. Am. 71, 1523-1528 (1981).

5. R. M. A. Azzam, "Extrema of the magnitude and the phase of a complex function of a real variable: application to attenuated internal reflection," J. Opt. Soc. Am. A 5, 11871192 (1988).

6. H. Li and S. Xie, "Measurement of the refractive index of biotissue by total internal reflection," Appl. Opt. 35, 17931795 (1996).

7. M.-H. Chiu, J.-Y. Lee, and D.-C. Su, "Refractive-index measurement based on the effects of total internal reflection and the uses of heterodyne interferometry," Appl. Opt. 36, 2936-2939 (1997).

8. S. G. Jennings, "Attenuated total reflectance measurements of the complex refractive index of polystyrene latex at $\mathrm{CO}_{2}$ laser wavelengths," J. Opt. Soc. Am. 71, 923-927 (1981).

9. R. G. Pinnick, S. G. Jennings, D. C. Boice, and J. P. Cruncleton, "Attenuated total reflectance measurements of the complex refractive index of kaolinite powder at $\mathrm{CO}_{2}$ laser wavelengths," Appl. Opt. 24, 3274-3285 (1985). 